\title{
THE CHARGES FOR ESRD TREATMENT OF DIABFTICS
}

\author{
Dean G. Smith, Linda C. Harlan ${ }^{2}$ and Victor M. Hawthorne ${ }^{2}$ \\ 'Department of Health Services, Management and Policy. School of Public Health, The University \\ of Michigan, Ann Arbor. MI 48109 and 'Department of Epidemiology, School of Public Health, \\ The University of Michigan, Ann Arbor, MI 48109. U.S.A.
}

(Recoiled in revised form 11 August 1988)

\begin{abstract}
To evaluate the differential charges for treating end-stage renal disease (ESRD) associated with diabetes mellitus, Medicare billing data are analyzed. The charges of 244 patients in the Michigan Kidney Registry identified as having (ESRD) from diabetes are compared with charges of 902 nondiabetic patients. Average annual charges for ESRD treatment for diabetics are $\$ 29,671( \pm 27,662)$ which are $\$ 4695( \pm 1344)$ higher than charges for nondiabetics. The majority of the difference $(84.3 \%)$ is attributable to higher inpatient hospital charges. Most of the remainder $(14.5 \%)$ is attributable to higher physician and medical supply charges. Charges for treatment of diabetics are higher on all modalities of treatment, but differences are not significant among modalities.
\end{abstract}

Charges Diabetes End-stage renal disease

\section{INTRODUCTION}

The treatment of end-stage renal disease (ESRD) in the United States consumes over $\$ 2.5$ billion every year [1]. Currently, diabetes mellitus represents the primary diagnosis for nearly one quarter of all patients on ESRD treatment [2]. In recent years the number of diabetics being accepted onto ESRD treatment programs has been increasing both in the U.S. and throughout Europe [3,4]. The European Dialysis and Transplant Registry reported an increase in the proportion of diabetics accepted from less than $0.5 \%$ in 1972 to $7.3 \%$ by 1981 [4]. This registry also reported diabetes to be the only primary cause of ESRD which is increasing in all industrialized countries [5]. Knowles has estimated that $50 \%$ of the patients with insulin dependent diabetes mellitus will progress to ESRD [6]. Clearly ESRD in diabetics is a major health concern which generates substantial medical costs.

Few previous studies have examined the cost of ESRD treatment for diabetic patients, and there are no studies which have examined the cost of ESRD treatment for diabetic patients by modality adjusting for patient characteristics. Beltzer et al. found that hospital costs for the year after a transplant were $\$ 1507$ and $\$ 2600$ higher for diabetics than nondiabetics receiving living-related and cadaveric transplants, respectively [7]. Costs associated with livingrelated transplants were found to be lower than costs associated with cadaver transplants for both diabetics and nondiabetics. Evans et al. adjusted for case-mix factors and found annual outpatient provider reimbursement to be $\$ 1646$ lower for diabetics than for nondiabetics, but physician reimbursement, inpatient and total reimbursement were $\$ 2868, \$ 5310$ and $\$ 6532$ higher, respectively [8]. Some reimbursement comparisons by modality were made in general by Evans et al., but not specifically for diabetics.

In this study we provide specific information on Medicare allowable charges for treatment of ESRD in diabetics and compare them to charges for treatment in nondiabetics, adjusting for selection and patient characteristics. We also examine these charges by treatment modality in an attempt to identify those treatment modalities which might be less expensive. If any modalities were found to be less expensive there would 
be potential for savings in the large expenditures for ESRD treatment in diabetics.

\section{MATERIALS AND METHODS}

Data for this study come from the Health Care Financing Administration (HCFA) through the Urban Institute. The sample was derived by requesting data on all ESRD patients in Michigan from 1982-1984 with onset of ESRD from 1981-1983, as identified by the Michigan Kidney Registry (MKR). The decision rule for admission to the sample was onset of ESRD. The aim, therefore, was to observe the entire population. An observation is defined as having complete information on a patient for a particular year, for however long the patient was alive and had ESRD during that year. There are between one and three obervations for each patient, depending on time of death, year of onset of ESRD, and data availability. However, there are no observations on some patients because of death in 1981 . Using the average rate of death for patients dying in 1982 and 1983 as their first year of ESRD, ony approximately 18 too few patients in sample of $2292,(0.8 \%)$ were included in the study. Therefore, the sample is only slightly biased in overrepresenting patients surviving their first year of ESRD. A matching of HCFA and MKR data resulted in a sample of 487 annual (calendar year) observations on 243 diabetic patients and 1805 annual observations on 903 nondiabetic patients. This represents a match rate of approximately $62 \%$ (1146 out of 1832) between the complete HCFA records and MKR data.

\section{Charges}

The HCFA data include charges for medical services from provider billing records, and modality and patient characteristics information from the Patient Management-Medical Information System (PMMIS). Charges are defined as Medicare allowable charges. These charges are the sum of the reimbursement amounts paid by Medicare to providers and the copayments and deductibles paid directly by the patient. Medicare reimbursement amounts account for slightly under $80 \%$ of total charges. This measure of charges does not include payments for services not covered by Medicare, such as private duty nurses and transportation to a dialysis center or outpatient facility. It is now known how charges which are not covered by
Medicare vary by modality or are different for diabetic and nondiabetic patients. The lack of this information is a potential limitation of this and any other study which uses only Medicare billing data as a measure of charges.

Medicare reimbursements for dialysis treatments and physician services are paid under Part B and are based on predetermined fixed rates which are identical for home and center dialysis. Hospital reimbursement is paid on the basis of diagnosis-related groups (DRGs) which also involve predetermined fixed rates. The extent to which diabetics incur larger levels of charges and reimbursements is, therefore, more closely associated with a greater number of visits to health care providers than to larger payment amounts per visit. Medicare allowable changes do not reflect any increased intensity of treatment due to diabetes, and may, therefore, lead to a conservative estimate of the additional cost of treating ESRD in diabetics.

\section{Modality}

The modalities of treatment were defined using the scheme of Smith and Wheeler [9]. This definition of modality is based on annual aggregation of quarterly records of actual treatments received and designed specifically for this type of analysis. Defining modality in this way permits a close correspondence between modality and charges. Alternative definitions of modality, such as initial treatment or primary treatment, may be useful for survival or other analyses, but not for studies which attempt to match charges and treatment. This definition of modality includes the following treatments: the use of single, well-defined dialysis treatments [incenter hemodialysis (CHD), in-center peritoneal dialysis (PERI), and at-home continuous ambulatory peritoneal dialysis (CAPDH)], combinations of dialysis treatments [continuous ambulatory peritoneal dialysis training (CAPDTR), and other combinations of treatments (OTHER)], and transplants from livingrelated donors (LTRAN) and cadaver donors (CTRAN). For some modalities, such as home hemodialysis and the simultaneous or sequential use of CHD and CAPD, there were too few observations on diabetics for separate analysis. These observations $(34,7 \%)$ were excluded from the analysis. For each observation, again, a complete set of information on a patient in a given year, there is only one modality. For patients receiving multiple forms of treatment, 
Table 1. Characteristics of diabetic ESRD patients by treatment modality

\begin{tabular}{|c|c|c|c|c|c|}
\hline \multirow[b]{2}{*}{ Variable } & \multicolumn{5}{|c|}{ Dialysis modality } \\
\hline & $\begin{array}{l}\text { Center } \\
\text { hemodialysis } \\
(\mathrm{CH})\end{array}$ & $\begin{array}{c}\text { Center } \\
\text { peritoneal dialysis } \\
(\text { PERI) }\end{array}$ & $\begin{array}{l}\text { Center/home } \\
\text { CAPD training } \\
\text { (CAPDTR) }\end{array}$ & $\begin{array}{l}\text { Home CAPD } \\
(\mathrm{CAPDH})\end{array}$ & $\begin{array}{c}\text { Other dialysis } \\
\text { combinations } \\
\text { (OTHER) }\end{array}$ \\
\hline Age (years) & 58.7 & 58.8 & 53.2 & 44.4 & 50.4 \\
\hline Race (\% non-white) & 43.7 & 36.8 & 10.3 & 13.3 & 21.4 \\
\hline Sex $(\%$ female $)$ & 56.3 & 73.7 & 74.4 & 66.7 & 71.4 \\
\hline Date (months) & 9.7 & 8.7 & 8.6 & 8.1 & 10.5 \\
\hline Time (years) & 2.0 & 1.9 & 2.0 & 1.7 & 1.8 \\
\hline Death ( $\%$ dying) & 18.9 & 26.3 & 28.2 & 33.3 & 14.3 \\
\hline $1982(\%$ observed $)$ & 14.7 & 5.3 & 0.0 & 40.0 & 28.6 \\
\hline 1984 (\% observed $)$ & 38.7 & 26.3 & 64.1 & 13.3 & 21.4 \\
\hline$N$-Diabetic ${ }^{\mathrm{a}}$ & 238 & 19 & 39 & 15 & 14 \\
\hline \multirow[t]{2}{*}{$N$-Non-diabetic ${ }^{b}$} & 868 & 50 & 147 & 75 & 86 \\
\hline & \multicolumn{5}{|c|}{ Transplant modality } \\
\hline Variable & $\begin{array}{c}\text { Living-related } \\
\text { donor } \\
\text { (LTRAN) }\end{array}$ & $\begin{array}{c}\text { Cadaver donor } \\
\text { (CTRAN) }\end{array}$ & $\begin{array}{c}\text { Failed } \\
\text { (FTRAN) }\end{array}$ & $\begin{array}{l}\text { Diabetic } \\
\text { weighted } \\
\text { average }^{c}\end{array}$ & $\begin{array}{c}\text { Non-diabetic } \\
\text { weighted } \\
\text { average }^{c}\end{array}$ \\
\hline Age (years) & 37.7 & 38.2 & 35.8 & 51.4 & 51.5 \\
\hline Race ( $\%$ non-white) & 22.2 & 16.7 & 10.4 & 29.8 & 32.1 \\
\hline Sex $(\%$ female $)$ & 20.0 & 38.9 & 31.0 & 51.6 & 44.0 \\
\hline Date (months) & 11.2 & 10.5 & 9.7 & 9.8 & 10.1 \\
\hline Time (years) & 2.2 & 1.9 & 2.2 & 2.0 & 2.0 \\
\hline Death ( $\%$ dying) & 2.2 & 5.6 & 27.6 & 17.9 & 10.7 \\
\hline $1982(\%$ observed $)$ & 20.0 & 22.2 & 24.1 & 16.2 & 15.8 \\
\hline $1984(\%$ observed $)$ & 40.0 & 38.9 & 27.6 & 38.4 & 40.6 \\
\hline$N$-Diabetic ${ }^{\mathrm{a}}$ & 45 & 54 & 29 & 487 & - \\
\hline$N$-Non-diabetic ${ }^{b}$ & 122 & 239 & 86 & - & 1805 \\
\hline
\end{tabular}

${ }^{a}$ The number of observations of diabetic patients upon which the average characteristics are calculated. ${ }^{6}$ The number of observations of non-diabetic patients. Average characteristics are not presented by modality for these patients. 'The weighted averages include the observations from the additional six modalities which do not include sufficient numbers of observations for cost estimation.

the combinations of treatments are defined as distinct modalities, such as OTHER.

The selection of a particular modality, while not deterministic, is not completely random. Patients along with their physicians selcet that modality which is believed to be the most appropriate form of treatment, given their condition, anticipated survival and quality-of-life. No formal criteria have been followed for modality selection, as has been suggested for cancer patients by Simes [10] and cadaver kidney recipients by Starzl et al. [11] but the data suggest systematic selection of modalities.

A description of the data for diabetic patients by modality and for the average of nondiabetic patients on all modalities is presented in Table 1 . The distribution of patients by modality is very similar for diabetics and nondiabetics making comparisons of average characteristics between the two groups reasonable. The diabetic ESRD patients were, on average, nearly the same age as the nondiabetics. Diabetics were somewhat more likely to be white and female than nondiabetics. Diabetics also had a higher mortality rate during the study period. Among diabetics the patients were evenly divided between the genders and nearly $30 \%$ were black. The type of diabetes (insulin dependent vs noninsulin dependent) was not known for individuals in the present study. For all patients, those receiving transplants were more likely to be younger, white and male than dialysis patients, and successful transplant recipients were less likely to die during the study period.

The other variables, number of months observed in a year (Date), time since onset of ESRD (Time), and a marker for the particular year of the observation $(1982,1984)$ also vary by modality, but their primary importance is in the allocation of charges.

This sample appears to have an unusually large number of observations of persons using CAPD training. Although CAPD training typically occurs over a period of days or weeks, there were many observations which listed CAPD training for much longer periods of time. This is due mostly to the unit of modality observation being a quarter. If any training 
sessions are provided during a quarter then the defined modality for reimbursement on HCFA records is CAPDTR.

\section{Estimation}

To adjust for modality selection and differences in patient characteristics, a two-part estimation technique is employed. Failure to account for selection would result in biased estimates of charges by modality [12]. In the first part, a self-selection probit model is estimated for each modality. Individual probit models for the selection of each modality are estimated using patient characteristics (age, sex, race, time since onset of ESRD) as determinants of selection. A potentially important limitation of this study is the lack of additional patient and physician data which might more exactly determine selection. It has been shown that there are patterns of physician treatment recommendations which are independent of the above listed patient characteristics [13].

The probit model yields predicted values which are used to construct additional variables, so-called inverse Mill's ratios, for each patient. The results from the first part, essentially, reflect the effects of the patient's characteristics on selection of a modality. In the second part, these ratios are used as covariates along with the other patient characteristics in a multivariate least squares regression of the logarithm of annual charges. Logged charges are used to avoid the problems associated with extreme values which are frequently encountered in health care charge data [14]. Observations of less than $\$ 500$ for more than one quarter of a year and more than $\$ 200,000$ are considered to be gross outliers and are not included in the sample. Inclusion of these outliers, however, only affects the variances of the final results and not the mean values.

Standardized values of patient characteristics for each modality are used in the estimated equations to determine the charges for ESRD treatment by modality. The standardized value for length of time of observation (Date) is 12 months and 1983 for year of observation. This approach is described in general by Maddala [15] and terms of specific examples by Lee and Trost [16].

This approach permits a consistent and asymptotically efficient method of estimated charges. Some limitations in the estimation procedures, however, should be noted. First, having an unequal number of observations per patient indicates a potential lack of efficiency. This was not a problem in this case, as separate equations estimated using aggregated patient data and weighted least squares yielded nearly identical results. We also found that in time-series estimations, patients' past charges were not significant predictors of current charges. These results might not be true in general for other researchers using this method and should always be investigated. Second, for some modalities, such as those involving training or a transition between treatments, for which a patient would usually only be in this state for a short period of time, annualization of charges may lead to deceiving results. Caution must, therefore, be used when comparing estimated charges between steady-state and transitional modalities of treatment. And finally, since charges are estimated in logs, estimated standard deviations are also in logs. Standard deviations are, therefore, log-linear rather than linear and simple confidence intervals do not have the usual meaning.

The statistical analysis was conducted using the Michigan Interactive Data Analysis System (MIDAS), a program capable of handling the probit and multivariate least squares regression analyses required by this two-part technique.

\section{RESULTS}

Table 2 presents estimates of a full year's charges for ESRD treatment by modality for diabetic and nondiabetic patients holding constant the patient characteristics of diabetic patients. The interpretation of these values is that the charges in each row represent the estimated annualized (12 months) charges assuming that the average age, sex, race, time since onset of ESRD and proportion dying are the same for diabetic and nondiabetic patients. The only factor explaining the difference between the two columns is the presence of diabetes vs some other disease causing ESRD. The differences between rows are not adjusted for patient characteristics.

On average, diabetics have annual charges of $\$ 29,671$, which are significantly higher $(\$ 4695$, $p<0.01$ ) than charges for nondiabetics. Although standard deviations do not have their usual meaning in the strict sense, as an approximation for reference, a confidence interval of \pm 1344 is associated with the additional charges for ESRD treatment of diabetics. This adjusted difference in charges is less than the observed 
Table 2. Annual charges adjusted for patient characteristics (1983 dollars)

\begin{tabular}{lrrr}
\hline Modality & Diabetics & Non-diabetics & Difference \\
\hline Dialysis & & & \\
CH & $\$ 27,463$ & $\$ 23,470$ & $\$ 3993$ \\
PERI & 26,486 & 22,529 & 3957 \\
CAPDTR & 17.879 & 18,408 & -529 \\
CAPDH & 29,435 & 22,753 & 6682 \\
OTHER & 40,779 & 28.342 & 12,437 \\
Year of & & & \\
Transplant & & & \\
LTRAN & 46.797 & 41.553 & 5244 \\
CTRAN & 61,493 & 42,074 & 19,419 \\
FTRAN & 63,670 & 58,672 & 4998 \\
Year After & & & \\
Transplant & & & \\
LTRAN & 4320 & 3836 & 484 \\
CTRAN & 8325 & 5696 & 2629 \\
FTRAN & 50.584 & 47.057 & 3527 \\
\hline Weighted average & $\$ 29.671$ & $\$ 24.976$ & $\$ 4.695^{*}$ \\
\hline Indicates a significant difference from $0, p<0.01$, two-tailed test. \\
Indicates a significant difference from $0, p<0.05$, two-tailed test.
\end{tabular}

difference in charges due mostly to the higher death rate of diabetics. The death rate of diabetics is much higher than the death rate for nondiabetics, and is associated with diabetes independently as well as with other patient characteristics. The higher death rate of diabetics implies a larger number of "censored" observations (observations of persons for less than a full year) for diabetics than for nondiabetics. Therefore, adjusted charges are relatively higher for diabetics than for nondiabetics. However, deaths are associated with very high medical charges, especially for ESRD patients [17]. The net effect of the adjustment for death rates leads to relatively higher adjusted charges for nondiabetics than for diabetics, and hence the adjusted $\$ 4695$ difference is less than the observed difference in charges.

Of the total difference in charges between diabetics and nondiabctics, the majority $(84.3 \%)$ is explained by differences in inpatient hospital charges. Differences in charges by source of charges are presented in Table 3. Almost all of the remaining difference $(14.5 \%)$ is explained by differences in charges for physician services and medical supplies. There is only a very small difference in charges for outpatient services.

Differences in annual charges among dialysis modalities were not significant, except for OTHER $(p<0.01)$ and CHD $(p<0.05)$. Although the differences in average annual charges are quite large for PERI and CAPDH, the variances were proportionally larger. This insignificance may be partially attributable to the small numbers of observations of diabetic patients on PERI and CAPDH. Charges were actually lower for diabetics using CAPDTR, a modality which is either an initial modality or an intermediate stage between use of CHD or PERI and CAPDH. Adding charges incurred during training to the charges for CAPDH yields total charges which are similar to those of CHD and PERI.

In both the year of a transplant and after the transplant, charges were higher for diabetics than for nondiabetics. Charges were signifi-

Table 3. Distribution of annual charges and percent of charges by source (1983 dollars)

\begin{tabular}{lccc}
\hline Source & Diabetics & Non-Diabetics & Difference \\
\hline Physician/supplies & 3976 & 3297 & 679 \\
& $(13.4 \%)$ & $(13.2 \%)$ & $(14.5 \%)$ \\
Outpatient & 13,945 & 13,887 & 58 \\
& $(47.0 \%)$ & $(55.6 \%)$ & $(1.2 \%)$ \\
Inpatient & 11.750 & 7792 & 3958 \\
& $(39.6 \%)$ & $(31.2 \%)$ & $(84.3 \%)$ \\
\hline Total & $\$ 29,671$ & $\$ 24,976$ & $\$ 4,695$ \\
\hline
\end{tabular}


Table 4. Annual charges adjusted for patient characteristics associated with each treatment modality (1983 dollars)

\begin{tabular}{lccc}
\hline Modality & \multicolumn{3}{c}{ Patient characteristics } \\
\cline { 2 - 4 } & $\begin{array}{c}\text { Center } \\
\text { hemodialysis } \\
\text { (CHD) }\end{array}$ & $\begin{array}{c}\text { Center } \\
\text { peritoneal dialysis } \\
\text { (PERI) }\end{array}$ & $\begin{array}{c}\text { Home CAPD } \\
\text { (CAPDH) }\end{array}$ \\
\hline CHD & 27,463 & 29,301 & 27,169 \\
PERI & 25,879 & 26,486 & 23,612 \\
CAPDH & 30,891 & 31,287 & 29,435 \\
\hline
\end{tabular}

andicates that no differences between amounts are significant, $p<0.05$, two-tailed test. ${ }^{b}$ Columns are based on average patient

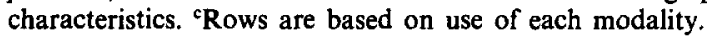

cantly higher for cadaver transplants in the year of a transplant. In the year after a transplant the differences in charges were smaller than the differences in the year of the transplant, and very small for living-related transplants. Differences in transplantation charges between diabetics and nondiabetics are similar in magnitude to differences in dialysis charges, although no two values are exactly the same.

Holding patient characteristics constant and adjusted for the selection of particular dialysis modalities reduced the difference in charges among modalities. These comparative annual charges for selected modalities and diabetics only are presented in Table 4 . The interpretation of these values is that the charges in each column represent the estimated annual charges assuming that the average age, sex, race, time since onset of ESRD and proportion dying are the same. The only factor explaining the difference between two columns is the use of the average patient characteristics of patients using a given modality. Similarly, the only factor explaining the difference between two rows is the use of one modality vs some other modality of treatment. For example, a patient currently using CHD (column 1) would be expected to incur charges of $\$ 27,463$ when using CHD (row 1), but only $\$ 25,879$ when using PERI (row 2).

\section{DISCUSSION}

The two questions addressed in this paper are: (1) are diabetic patients with ESRD more expensive to treat than nondiabetic patients; and (2) are there cost differences among treatment modalities that could be used to help control costs. The answers, briefly, are yes and probably no, repectively.

For most treatment modalities, annual charges were higher for diabetics than nondia- betics, but many differences were insignificant. Overall, charges for diabetics were $\$ 4695$ more per year than charges for nondiabetics. Of these additional charges, the majority were for inpatient services and the remainder for physician services and medical supplies. As suggested above, this observation is partially attributable to Medicare's reimbursement system. While there is no necessary reason for higher charges for hospitalization, physical services and supplies of diabetics to be associated with higher costs for dialysis treatments (the bulk of outpatient charges), there is no clear reason for costs to be nearly identical for diabetics and nondiabetics. It is expected, that the inpatient, physicial and supply charges reflect differences in the complexity and quantity of treatments for ESRD in diabetics which are not reflected in the charges for outpatient services because of the system of reimbursement. The actual difference in resources used to treat diabetics as compared to nondiabetics is probably underestimated by the difference in charges.

For diabetic patients electing dialysis treatments for ESRD, the costs are very similar among modalities, adjusting for patient characteristics and selection. Table 4 suggests that there may be some potential for cost savings by encouraging diabetic patients on CHD or CAPD to use PERI, but the expected savings are statistically insignificant and could be outweighed if there are costs or complications involved with changing modalities. While it appears that PERI is the cheapest form of treatment, a result which might not be expected, we regretfully do not have any insights to explain this result. There may be individual diabetic patients whose characteristics more closely match those of patients using other modalities which, on average, are less expensive. However, having the average patient using each dialysis modality change modality to one which 
is, on average, less expensive would not yield cost savings. This result is also influenced by the Medicare payment system which explicitly sets equal payment rates for home and center dialysis. A measure of the real cost of resources consumed by patients on each modality might yield different results. However, no such measure is available. Given the lack of significant differences in charges, decisions on dialysis modality selection should, and most likely already are, based on other considerations such as expected survival and quality-of-life.

In both the year of a transplant and after the transplant, charges were higher for diabetics than for nondiabetics. However, this does not suggest that transplants are not a cost-effective modality of treatment for diabetics. While the charges are higher for transplants in diabetics, the cost saving realized by a successful transplant are also higher. For example, if a patient on CHD were to receive a transplant from a cadaver donor, the savings in each year following a successful transplant would by $\$ 17,774$ $(\$ 23,470-5696)$ for the nondiabetic and $\$ 19,138$ $(\$ 27,463-8325)$ for the diabetic. In both cases the additional charge for the transplant would be paid back in terms of future saving within 5 years. The time to payback is nearly identical for diabetic and nondiabetic patients receiving transplants from either cadaver or living-related donors, even when probability of failure is included. The question of cost-effectiveness, or relative cost-effectiveness ultimately rests on the issue of expected survival after transplantation. The crude death rate in this sample and evidence from other studies suggests that survival is lower for diabetics than nondiabetics [18].

The next question which naturally arises is whether more diabetic patients should receive transplants. This analysis would suggest the answer is yes, but it is more difficult to evaluate for at least two reasons. First, suitable kidneys for transplantation are scarce and, therefore, transplant patients routinely receive extensive evaluations prior to becoming transplant candidates. Acceptance or rejection is frequently based on the outcome of coronary angiography, overall health status and age. A comparison of the relative health of diabetics vs patients with hypertension and other diseases is necessary to fully answer this question.

And second, as a result of the screening, patients accepted for transplantation are healthier and are less likely to die than dialysis patients. Therefore, the most cost-effective transplants may be those currently being performed, although this is not necessarily true in every case. When Khauli compared a group of diabetics who received transplants to a group of transplant candidates, for whom no acceptable kidney could be found, no significant difference in survival was seen in 24 months, although the trend towards better survival of those transplanted was beginning to emerge [19].

Although these results present a more detailed view of ESRD charges for diabetics than has been previously available, three cautions in their use are necessary. First, the data did not take into consideration the many individual characteristics of the patient's medical, social and emotional status. Some of these characteristics may be very important in terms of treatment selection and the ultimate success of a particular modality. Second, measures of improvement of stabilization of retinopathy, neuropathy or quality-of-life were not available for this analysis. These too may affect the results presented. And third, total charges for patient care were used rather than the marginal costs of ESRD treatment alone. Available data do not permit the separation of charges associated with ESRD, diabetes and other conditions. It is expected that a measure of resource costs would yield a larger difference between the costs of ESRD treatment for diabetics and nondiabetics. It is also possible that certain modalities are truely less expensive than others for particular patients, but that the use of medical services for other conditions and the nature of the reimbursement system did not allow these advantages to be observed. Not having found these less expensive modalities means that potential opportunities for saving may be missed.

Acknowledgements - The authors are grateful for the data provided by Dr Philip Held and the Urban Institute, for computer assistance from Ms Sue Eshleman, and for helpful comments from two anonymous reviewers. This work was supported by the Health Care Financing Administration (Grant no. 14-P-98372/5-04) and The University of Michigan, Diabetes Research and Training Grant (Grant no. DK 67445-05).

\section{REFERENCES}

1. Gornick M, Greenherg JN, Eggers PW and Dobson A Twenty years of Medicare: covered populations. use of benefits. and program expenditures. Hith Care Fin Rev 1985; 7: 22-55

2. Health Care Financing Administration. End-Stage Renal Disease Patient Profile Tables. Baltimore. MD Department of Health and Human Services, HCFA. Bureau of Data Management and Strategy; 1984. 
3. Rosansky SJ and Eggers PW. Trends in the U.S. end-stage renal disease population: 1973-1983. Am J Kid Dis 1987; 9: 91-97.

4. Jacobs C, Brunner FP, Brynger $\mathrm{H}$. et al. The first five thousand diabetics treated by dialysis and transplantation in europe. Diab Neph 1983; 2: 12-16.

5. Keen $H$ and Legrain $M$. Prevention and Treatment of Diabetic Nephropathy. Lancaster, England: MTP Press; 1983.

6. Knowles HC. Magnitude of renal failure problem in diabetic paticnts. Kidney Int 1974; 6: 2-10.

7. Beltzer FO, Miller DT, Sollinger $\mathrm{H}$ et al. Renal transplantation-a view of the 1980s. Semin Nephrol 1983; 2: $99 \cdot 110$.

8. Evans RW, Manninen DL, Hart LG, Garrison LP. The Treatment of End-Stage Renal Disease in the U.S.; Selected findings from the National Kidney Dialysis and Kidney Transplant Study. Seattle, WA: Battelle Human Affairs Research Center; 1984.

9. Smith DG and Wheeler JRC. Switching and the definition of modality in end-stage renal disease treatment. Med Care 1987; 25: 1168-1178.

10. Simes RJ. Treatment selection for cancer patients application of statistical decision theory to the treatment of advanced ovarian cancer. J Chron Dis 1985; 38 : 171-186.

11. Starzl TE et al. A multifactorial system for equitable selection of cadaver kidney recipients. JAMA 1987; 257: 3073-3075.
12. Heckman J. Sample selection bias as a specification error. Econometrica 1979; 47: 239-267.

13. Deber RB, Blinder IN, Carr LM, Barnsley JM. The Impact of Selected Patient Characteristics on Practitioners' Treatment Recommendations for End-Stage Renal Disease. Med Care 1985; 23: 95-109.

14. Welch WP, Frank RG, Diehr P. Health care costs in health maintenance organizations: correcting for selfselection. In: Scheffler RM, Rossiter L, Eds. Advances in Health Economics and Health Services Research, Vol 5; 1984: 95. 128.

15. Maddala GS. Limited-Dependent and Qualitative Variables in Econometrics. New York: Cambridge University Press; 1983.

16. Lee LF and Trost RP. Technical training and earnings: a polychotomous choice model with selectivity. Rev Econ Stat 1984; 66: 151-156.

17. Eggers PW, Connerton R, McMullan M. The medicare experience with end-stage renal disease: trends in incidence, prevalence, and survival. Hith Care Fin Rev 1984; 5: 69-88.

18. McCall $\mathbf{N}$. Utilization and costs of medicare services by beneficiaries in their last year of life. Med Care 1984; 22: 329-342.

19. Khauli RB, Novick AC, Steinmuller DK et al. Patient survival and rehabilitation of diabetics with end-stage renal disease: comparison of theraputic modalities. Trans Proc 1985; 17: 178-181. 